\title{
Evaluating Three-dimensional Effects on the Behavior of Compliant Bistable Micromechanisms
}

\author{
Brian B. Cherry \\ Larry L. Howell \\ Ihowell@byu.edu \\ Brian D. Jensen
}

Follow this and additional works at: https://scholarsarchive.byu.edu/facpub

Part of the Mechanical Engineering Commons

\section{Original Publication Citation}

Cherry, B.B., Howell, L.L., and Jensen, B.D., "Evaluating Three-dimensional Effects on the Behavior of Compliant Bistable Micromechanisms," Journal of Micromechanics and Microengineering, Vol. 18, 951-1 to 951-1, 28.

\section{BYU ScholarsArchive Citation}

Cherry, Brian B.; Howell, Larry L.; and Jensen, Brian D., "Evaluating Three-dimensional Effects on the Behavior of Compliant Bistable Micromechanisms" (2008). Faculty Publications. 171.

https://scholarsarchive.byu.edu/facpub/171 


\title{
Evaluating Three-Dimensional Effects on the Behavior of Compliant Bistable Micromechanisms
}

\author{
Brian B. Cherry, Larry L. Howell, Brian D. Jensen \\ Department of Mechanical Engineering, Brigham Young University, Provo, Utah \\ 84602, U.S.A. \\ E-mail: lhowell@byu.edu
}

\begin{abstract}
.
Fully compliant bistable micromechanisms (FCBMs) have potential use in numerous applications, including switches, relays, shutters, and low-power sensing arrays. Two-dimensional finite element models for these FCBMs have been used in device analysis and design, and provided an adequate match to preliminary experimental data. However, with more extensive experimentation over a large range of designs, some results proved to be radically different than predicted, with trends not consistent with effects such as stiction or electrostatic forces. Two different types of behavior, Behavior 1 and Behavior 2, are observed and explained, only one of which is predicted by 2-D models. This paper tests the hypothesis that three-dimensional effects can dramatically influence the motion characteristics of FCBMs. Three-dimensional finite element models were constructed, compared to 2-D models, and validated for the purpose of testing the hypothesis. Off-axis and eccentric loads are shown to cause behavior consistent with experimental data for Behavior 2.
\end{abstract}

Submitted to: Journal of Micromechanics and Microengineering

\section{Introduction}

Compliant micromechanisms obtain some of their mobility from the deflection of flexible members rather than traditional pin joints and springs. Bistable mechanisms are devices that tend to remain in one of two stable equilibrium positions [1]. Fully Compliant Bistable Micromechanisms (FCBMs) 2] are devices that incorporate these two characteristics. Compliant bistable MEMS have potential use in numerous applications, including switches [3], relays [4, 5, 6], shutters [7, crash sensors [8, latchup testing [9, micro-positioning [10, and micro-shutter positioning [11. FCBMs show promise as threshold sensors where the device snaps between positions after the occurrence of a prescribed event, such as a certain acceleration magnitude [12. The necessary switching power is obtained from the phenomenon being sensed, and input power is only required to read or reset the sensor. Modeling of bistable mechanisms [13, 14, 15, 16] is important for determining the performance of the devices and the nonlinearities associated with the behavior makes them too complex to design by trial and error approaches.

As this paper will show, experimental data for FCBMs designed for various applications exhibited unexpected inconsistencies. It was even possible for the 


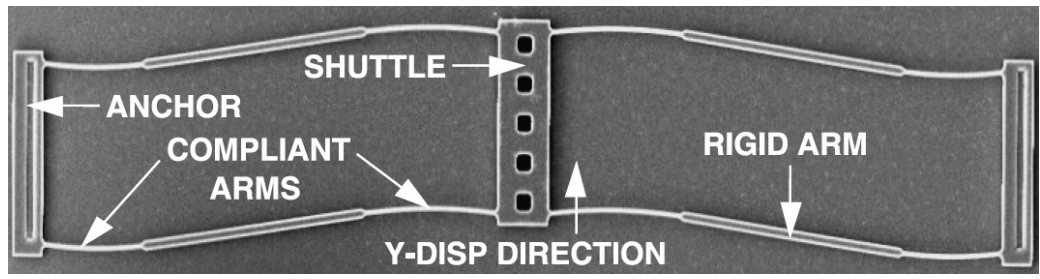

Figure 1. The FCBM (Bistable-A) components (SEM).

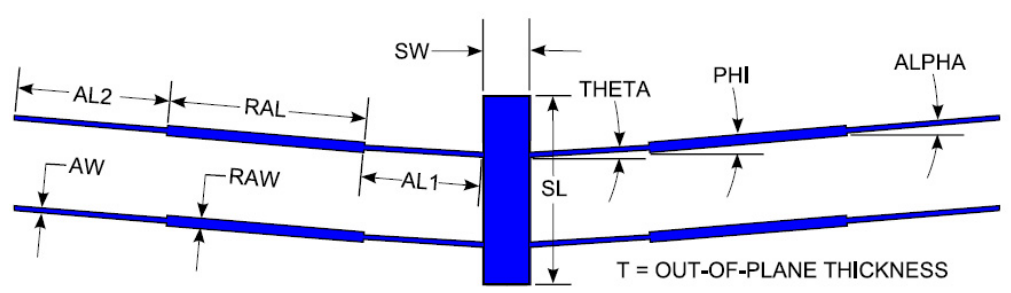

Figure 2. Dimensions of the bistable mechanism.

same device to exhibit radically different behavior at different times. For example, some devices did not have bistable behavior when the analysis predicted that they should be bistable. It was hypothesized that these differences could be the result of three-dimensional motions (such as out-of-plane rotation and displacement) that were not considered by 2-D models. Two-dimensional finite element analysis (FEA) models have been used to design and analyze FCBMs [17. Device symmetry, model simplicity, and the consistent predictions of preliminary experimental results were justifications for using the 2-D models. Quick convergence of the simplified models is particularly important when optimizing the design to meet specified design criteria, particularly considering the non-linearities and instabilities that slow convergence in FCBM analysis. In this paper, detailed 3-D models of FCBMs were created to test the hypothesis that 3-D effects can have a dramatic influence on the motion characteristics of FCBMs. Diagnostic test devices were fabricated and tested to gather experimental data for model validation. The results show that out-of-plane and off-axis loads can significantly affect the behavior of FCBMs.

\section{Fully Compliant Bistable Mechanisms}

A scanning electron micrograph of the FCBM (in the second stable equilibrium position) components is shown in Figure 1. Figure 2 shows the variables important for the parametric design of an FCBM. Variables include rigid arm length $(R A L)$, arm width $(A W)$, rigid arm width $(R A W)$, compliant arm length $1(A L 1)$, compliant arm length $2(A L 2)$, the angle of arm length 1 (THETA), the angle of the rigid arm length $(P H I)$, the angle of arm length $2(A L P H A)$, the out-of-plane thickness $(T)$, the shuttle width $(S W)$, and the shuttle length $(S L)$. The FCBM relies on the deflection of the compliant arms to move to its alternate stable position. YDISP is the actuated shuttle displacement. The Young's Modulus $(E X)$, Poisson's ratio $(P R)$, and device thickness are considered constant for a given device.

A nominal design, referred to as "Bistable-A", was selected as a benchmark for 
Table 1. Bistable-A design

\begin{tabular}{|c|c|}
\hline Variable & Magnitude \\
\hline$A L 1(\mu \mathrm{m})$ & 75.7 \\
\hline$A L 2(\mu \mathrm{m})$ & 57.3 \\
\hline$R A L(\mu \mathrm{m})$ & 126.3 \\
\hline$A W(\mu \mathrm{m})$ & 2.5 \\
\hline$R A W(\mu \mathrm{m})$ & 5.2 \\
\hline$T H E T A\left(^{\circ}\right)$ & 6.4 \\
\hline$P H I\left(^{\circ}\right)$ & 5.6 \\
\hline$A L P H A\left(^{\circ}\right)$ & 6.6 \\
\hline$T(\mu \mathrm{m})$ & 3.5 \\
\hline$E X(\mathrm{GPa})$ & 164 \\
\hline$P R$ & 0.22 \\
\hline$Y D I S P(\mu \mathrm{m})$ & 46 \\
\hline
\end{tabular}

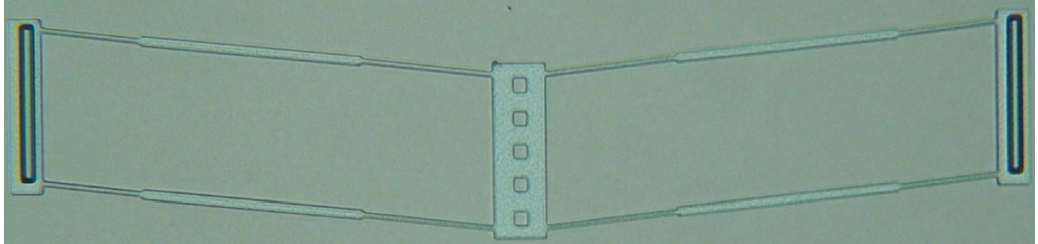

(a) First stable equilibrium position

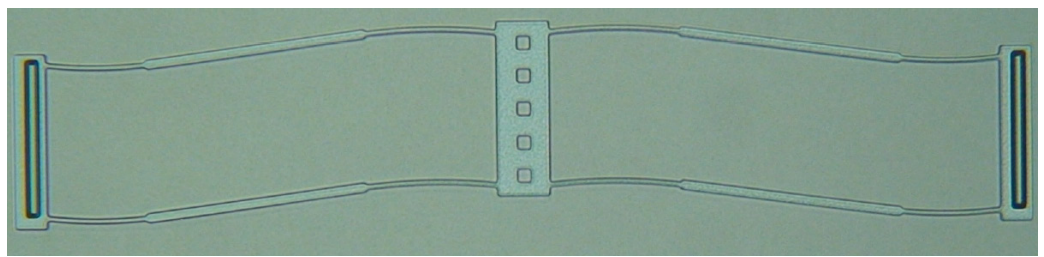

(b) Second stable equilibrium position

Figure 3. Bistable-A design manufactured with MUMPs [18.

variable changes. The nominal dimensions are listed in Table 1. This design was chosen because it demonstrated good bistable characteristics [12]. It has two distinct stable equilibrium positions, its stresses did not exceed critical stress levels, and the corresponding finite element models were well behaved. Figure 3(a) displays this device in the first stable position, and the second stable equilibrium position is shown in Figure 3(b),

The force-displacement relationship for Bistable-A, which is typical of other FCBM designs, is shown in Figure 4 (the plot is based on results predicted by the finite element model described later, with parameters described in Table 1). The device has two stable equilibrium positions and an unstable equilibrium position which occur at points where there is zero reaction force on the force-displacement curve. $F_{\max }$ is the force that must be applied to displace the mechanism from the first to the second stable equilibrium position, while $F_{\min }$ is the force that must be applied to displace the mechanism from the second to the first stable equilibrium position. The 


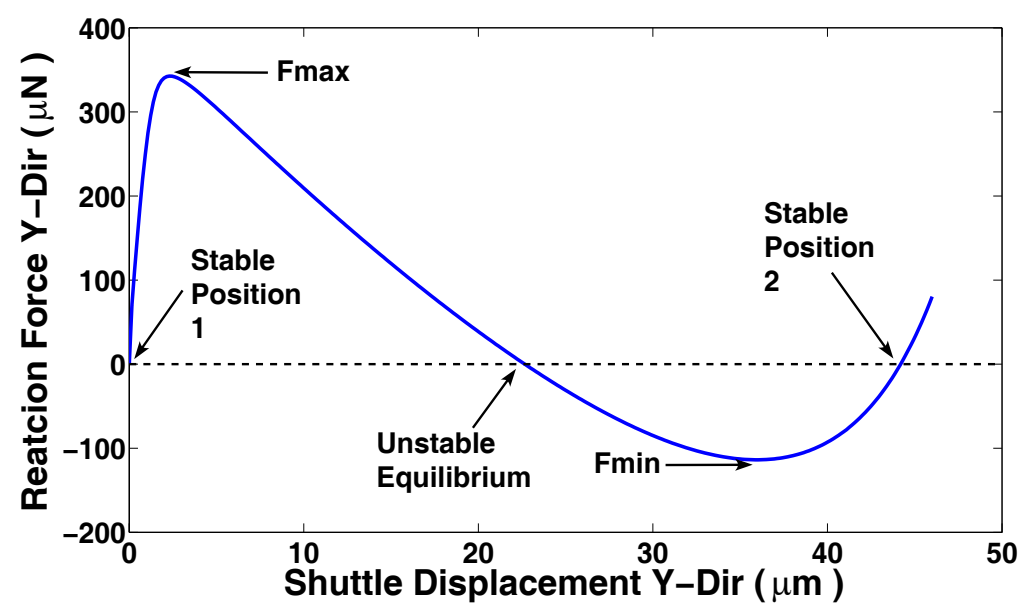

Figure 4. Force-displacement plot for Bistable-A design.

actuation approach used with the mechanism can influence its boundary conditions and can influence the resulting 3D effects. Thermal actuation [17, piezoelectric effects [15, 16, and inertial effects on the shuttle [12] have been proposed to switch bistable mechanisms. The work in this paper provides insight for other actuation approaches, but the boundary conditions are made to be consistent with a threshold accelerometer where an inertial load causes the switch between positions.

\section{Experimental Results}

Reaction force and displacement data were collected for two FCBMs, each manufactured in the Multi-User MEMS Process (MUMPs) [18 on a separate die. The geometry of the FCBMs matched the Bistable-A device listed in Table 1 . The first die was chemically released, then tested approximately twenty-four hours later. This is referred to as Device 1. The second die was released separately, and the FCBM was tested immediately afterward (Device 2). It could be assumed that both Device 1 and Device 2 had the same geometry and material property values.

A force gauge and probe guide was attached to the FCBM shuttle to retrieve all necessary reaction force and displacement data, as shown in Figure 5 The force measurement system affects the device boundary conditions, and the set up was designed to minimize the influence of the measurement system on the performance. To retrieve the force-displacement data, the tip of a probe was placed in the probe guide, and moved in the direction necessary to cause a transition between stable equilibrium positions [19. The force gauge was customized for the expected magnitudes of force and displacement it would experience while retrieving data from the theoretical forcedisplacement plot. Because the probe guide and force gauge are rigidly attached, one end of the force gauge is displaced the same amount as the probe guide. The side of the force gauge attached to the FCBM shuttle is then displaced a magnitude that is dependent on the stiffness of the force gauge. A vernier designed to provide displacement values for each side of the force gauge is recorded with a video camera through a microscope, and the displacements are retrieved from the vernier. By 


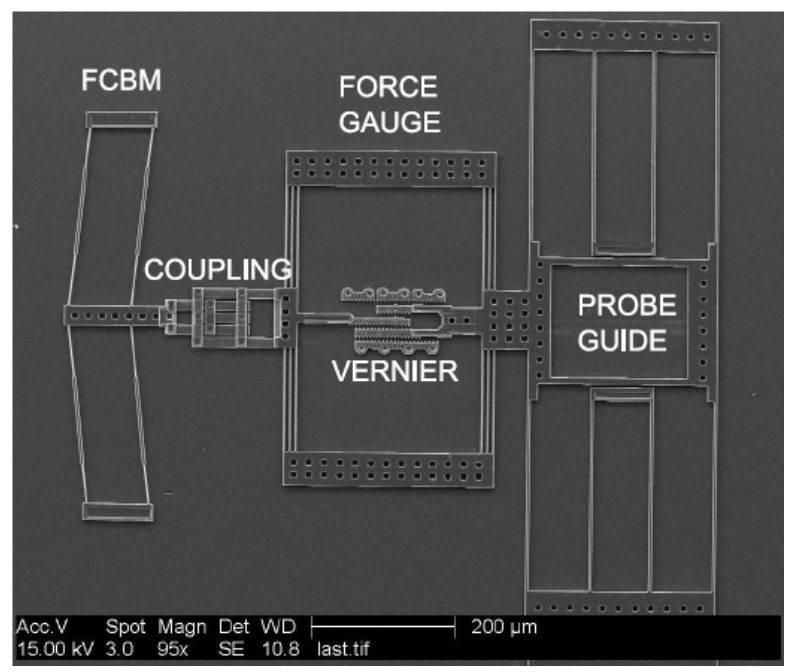

Figure 5. Test device used in MUMPs experimental data comparison (SEM).

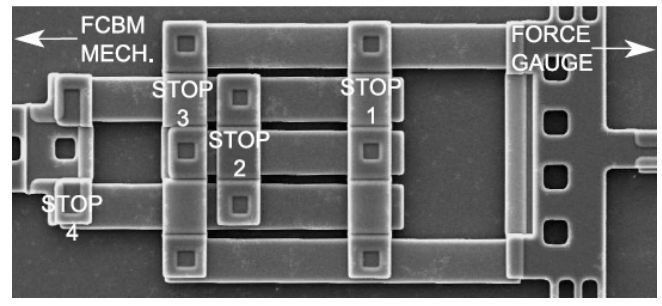

Figure 6. The coupler mechanism (SEM). This is labeled as the "COUPLING" in Figure 5

knowing the stiffness and displacement of each end of the force gauge, the reaction forces were determined.

A coupling between the force gauge and FCBM shuttle was designed to allow the capture of force data between the stable equilibrium positions. The coupling mechanism was constructed with beams that translated parallel to each other, as shown in Figure 6. Three of the beams are rigidly attached to the force gauge, while two beams are rigidly attached to the FCBM shuttle. Stops 1 and 3 are rigidly attached to the force gauge, and stops 2 and 4 are attached to the FCBM shuttle. As the FCBM is pulled from the first stable equilibrium position to the second, stop 3 pulls stop 2 to the right. As the force gauge pushes the FCBM shuttle back into the first stable equilibrium position, stop 3 pushes stop 4 to the left. The distance between each stop allows the FCBM to freely transition to its alternate stable position relatively unrestrained. Including the coupling mechanism reduced the constraint of the force gauge on the FCBM. The coupling design also allowed the FCBM shuttle some freedom to roll, pitch, and yaw.

Figure 7 shows the experimental results for Device 1 and Device 2, as well as the predicted results from the 2-D plane element model, which will be described further in the next section. The data labeled "Device 1: 1-2" and "Device 1: 2-1" were retrieved from the transition from the first to second and second to first stable equilibrium 


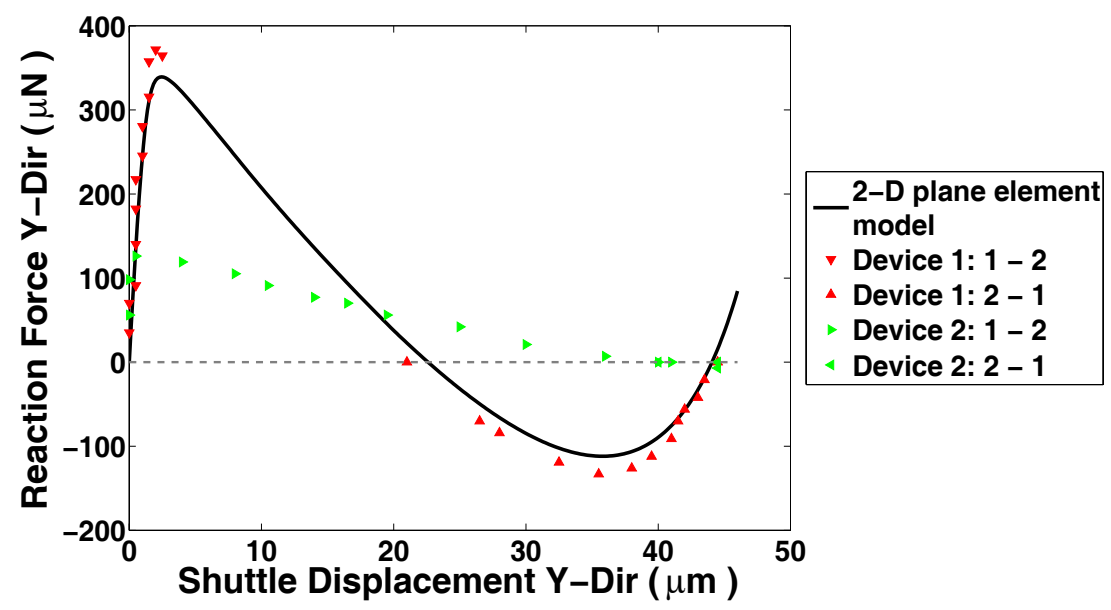

Figure 7. Experimental data collected from Device 1 and Device 2.

positions of Device 1, respectively. These experimental results were expected, as can be seen by the 2-D model prediction. The data labeled "Device 2: 1-2" and "Device 2: 2-1" are the experimental results for Device 2. Although Device 1 and Device 2 are essentially identical, their force-displacement plots are radically different. The peak reaction force for Device 2 is only a third of that for Device 1 . Device 2 is also barely bistable, as evidenced by the very small experimental $F_{\text {min }}$ value. The unexpected behavior of Device 2 is not accounted for by stiction or similar phenomena because the trends are opposite of what would be predicted for those behaviors (i.e. the reaction forces would increase to account for both the elastic energy stored in the flexible beams and friction). Further, it was possible for Device 1 to undergo a behavior similar to that shown here for Device 2 , and vice-versa. It was hypothesized that the large difference in behavior is due to three-dimensional effects that had a significant influence on the FCBM behavior, and was not accounted for in the 2-D model. To test this hypothesis, three-dimensional models were created with the goal of predicting and understanding both behavior conditions.

\section{FEA Models of the FCBM}

Several FEA models of the FCBM were created and then compared to each other and to experimental results. This information was used to evaluate the applicability of the 2-D models and to test the hypothesis that 3-D effects can have a significant influence on FCBM behavior. The models are described below. (See 20] for more details on the models and eccentric loading conditions [21.)

\subsection{Two-Dimensional Models}

4.1.1. 2-D Plane Element Model. A 2-D model was created that included all four compliant legs and the shuttle, as shown in Figure 8(a). This model was used in this work for comparison to other models. Displacement is applied at a location on the front face of the shuttle, and the resulting reaction forces are recorded at each displacement value. Zero-displacement boundary conditions were assigned to simulate the anchors. 


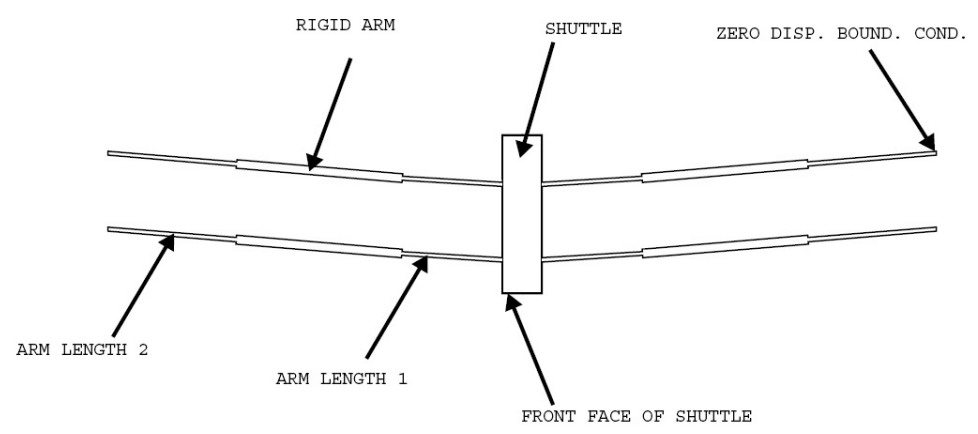

(a) 2-D plane element model with approximately 19,500 elements and 42,000 nodes

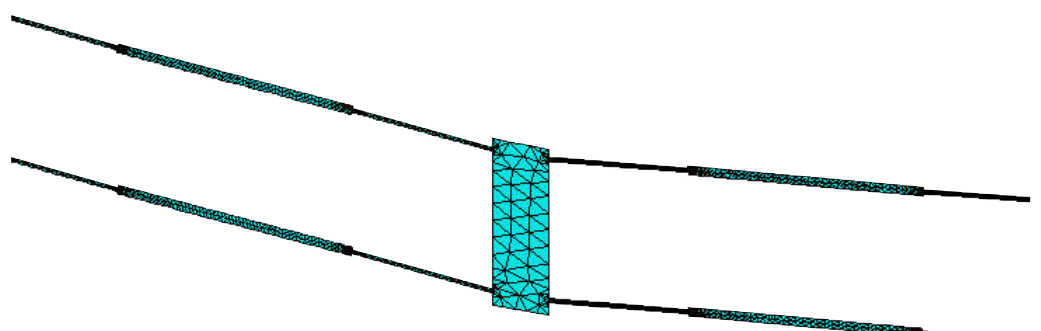

(b) Eight-node quad-element model with approximately 2,500 elements and 6,200 nodes

Figure 8. 2-D models.

The model was created using 4-node plane elements capable of large-deflection analysis (ANSYS plane2 elements set for plane stress conditions with a defined thickness), and the mesh contained approximately 19,500 elements and 42,000 nodes.

4.1.2. 2-D 8-Node Quad Element Model. A fully parametric model was constructed with eight-node quad-elements (ANSYS plane82 elements) to provide more accurate results for automated meshes, and to tolerate irregular shapes without much loss of accuracy (Figure 8(b)p. The elements can tolerate large deflection and large strain. The Bistable-A model resulted in approximately 2,500 elements and 6,200 nodes. Displacement was applied to each node on the face of the shuttle, and the reaction force was retrieved as a sum over the nodes on that face. This displacement application method constrained the shuttle to symmetric displacement.

\subsection{Three-Dimensional Models}

4.2.1. 3-D Beam Element Model. A simplified 3-D beam element model (ANSYS beam4 element) was created (Figure 9(a)p. The model is fully parametric, allowing for alternate geometries, material properties, shuttle displacement values, and mesh refinement. To assist with validation of the three-dimensional FCBM characteristics found by the solid element model discussed next, critical node locations were made available for displacement and applied off-axis forces.

An element was chosen with tension, compression, torsion, and bending 


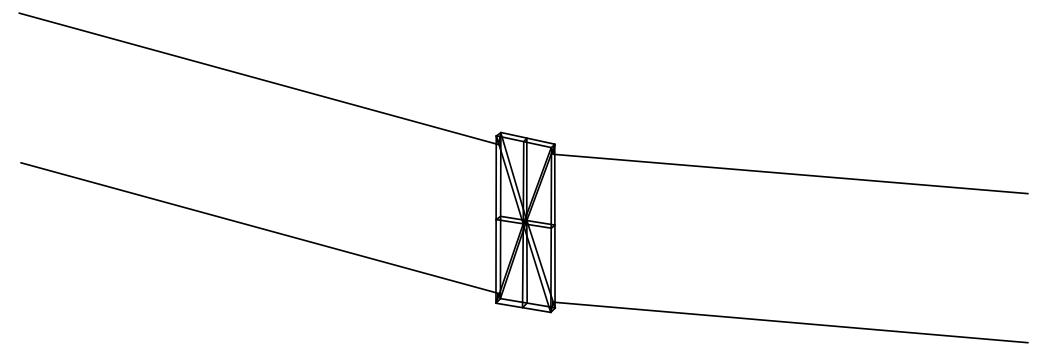

(a) 3-D beam element model with approximately 11,600 elements and 11,600 nodes

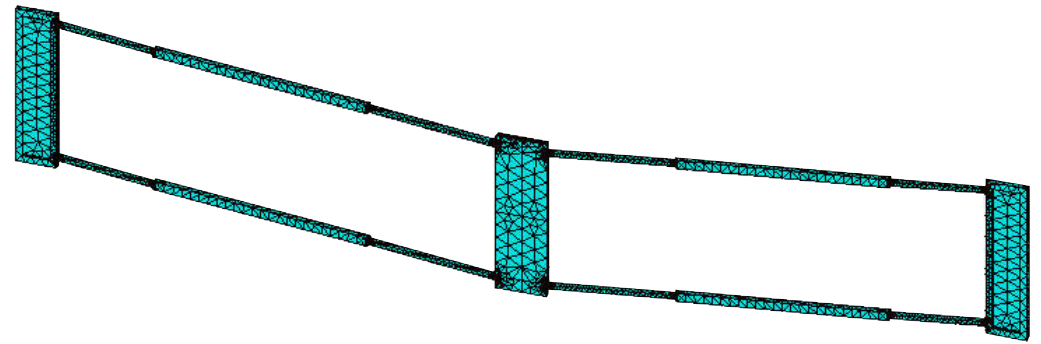

(b) 3-D solid element model with approximately 21,000 elements and 40,000 nodes

Figure 9. 3-D models.

capabilities (ANSYS beam4 element). This element is also capable of dealing with stress stiffening and large strain. To maintain rigidity of the shuttle and rigid arms, the elements were assigned a cross-sectional area and moment of inertia using the dimensions provided in Table 1 . Zero displacement boundary conditions were applied at the ends of the compliant arms connected to the anchors. A refined mesh was used in the compliant arm sections, a moderately coarse mesh was assigned to the rigid arms, and a coarse mesh was assigned to the shuttle. The model resulted in approximately 11,600 elements and 11,600 nodes, and converged to a solution in approximately two hours.

4.2.2. 3-D Solid Element Model. A more complete, 3-D model was created using 3 -D solid elements (ANSYS solid95 elements) with the intent of recovering the threedimensional characteristic of the FCBMs with a high level of accuracy (Figure 9(b)]. The model requires approximately nine hours to converge to a solution due to a large number of nodes and elements (approximately 21,000 elements and 40,000 nodes). This model incorporated geometric detail that simplified models did not provide. It is fully parametric, allowing for alternate geometries, material properties, and shuttle displacement values. The level of mesh refinement is also parametrically controlled for ease of parallel processing, and mesh optimization approaches were employed to efficiently refine the mesh [20].

One geometric detail included in the model was fillets at each of the compliant arm/rigid arm, compliant arm/shuttle, and compliant arm/anchor intersections. When the bistable mechanisms are produced, fillets naturally form at the intersection of two faces. Figure 10(a) shows a scanning electron micrograph (SEM) of a compliant arm/anchor interface fillet. 


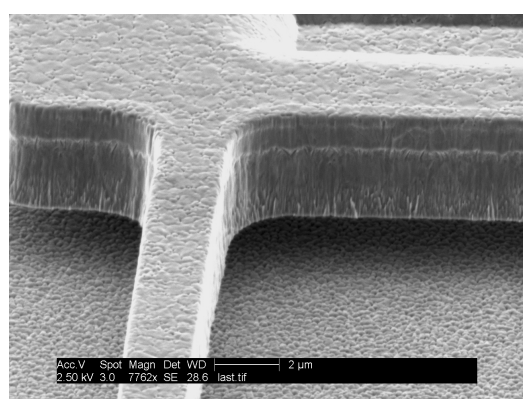

(a) SEM of anchor and fillets

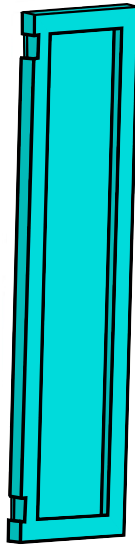

(b) Top

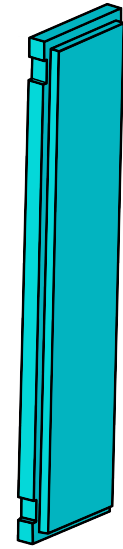

(c) Bot.

Figure 10. FCBM anchors.

Anchor details were also included in the 3-D solid element model. When the anchors are manufactured, a recess exists in the top surface, as can be seen in the 3 -D model of the anchor (Figure 10(b)) . The area contacting the substrate on the bottom of the anchor is equal in size to this recess. The bottom view of the anchor in Figure 10(c) shows the resulting overhanging edge. Figure 10(a) displays an SEM of the compliant arm/anchor intersection and the clearance between the overhanging edge and the substrate. The recess in the top of the anchor can also be seen in the upper-right corner. While the 2-D plane element model prescribes zero-displacement boundary conditions at the outer edges of the compliant arms, the 3-D solid element model prescribes zero-displacement boundary conditions at the entire bottom face of the anchor. Any bending that might occur at the overhanging edge of the compliant arm/anchor intersection may affect the behavior of the FCBM.

The 3-D solid element model also accounts for residual stress. Manufacturing the FCBMs using surface micromachining methods can create a uniform residual stress throughout the mechanism [22, 23, 24]. For example, FCBMs manufactured using MUMPs exhibit a negative or compressive residual stress that causes each of the four arms to slightly lengthen.

\subsection{Comparison of Models}

While the 2-D models have the ability to quickly retrieve the force-displacement data for an FCBM, they lack the ability to recover three-dimensional behavior. The 3-D models were used extensively to recover this three-dimensional data, but were first compared to the 2-D models. The Bistable-A design (Figure 1 and Table 1) was used in the comparison of models. The same number of loadsteps was used on each device to displace the FCBM shuttle $46.0 \mu \mathrm{m}$.

All four models converged to approximately the same stable and unstable equilibrium positions. However, Figure 11, shows that the 2-D models produced forcedisplacement plots that slightly differed from those predicted by the 3-D models. Table 2 lists the $F_{\max }$ and $F_{\min }$ values calculated by each of the four models. The 


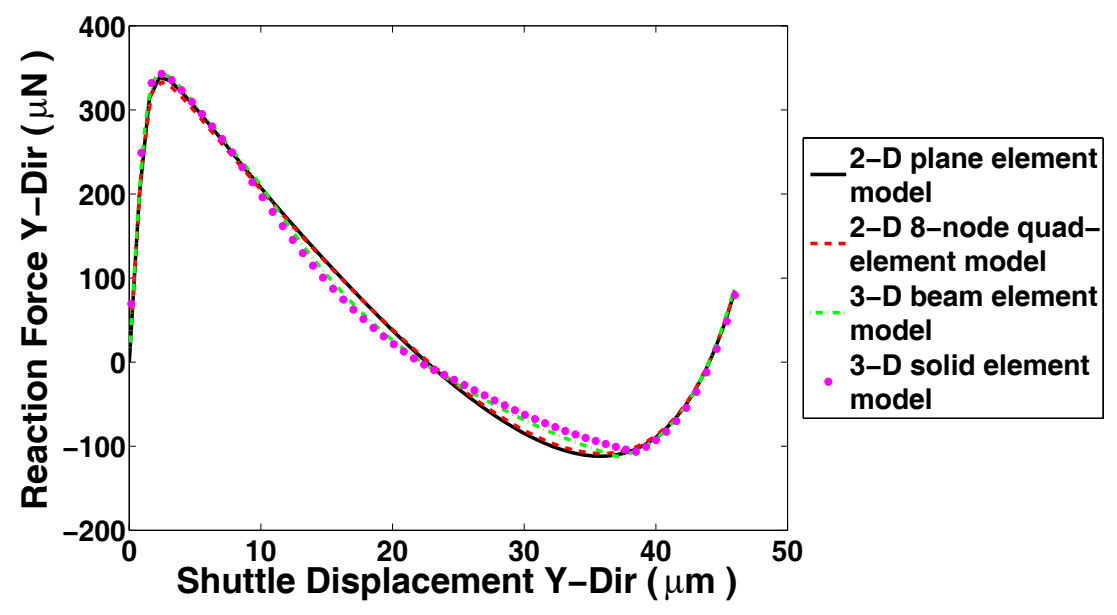

Figure 11. Force-displacement comparison of four models.

Table 2. Model comparison of force-displacement values.

\begin{tabular}{|c|c|c|}
\hline Model & $F_{\max }(\mu N)$ & $F_{\min }(\mu N)$ \\
\hline 2-D PLANE ELEMENT & 339.3 & -111.9 \\
\hline 2-D QUAD ELEMENT & 333.2 & -108.8 \\
\hline 3-D BEAM ELEMENT & 343.1 & -112.2 \\
\hline 3-D SOLID ELEMENT & 343.1 & -106.4 \\
\hline
\end{tabular}

difference in the general shape of the force-displacement plot between the 2-D and 3 -D models suggests that the 3 -D models are predicting characteristics that the $2-\mathrm{D}$ models are not.

\subsection{Validation of Models}

The models were validated by comparing the finite element predictions to experimental data. FCBMs were designed, fabricated, and tested. These devices, their results, and the comparison to predicted results are described next.

The FCBM geometry listed in Table 3 was created using Sandia National Laboratory's SUMMiT V manufacturing process 25. Reaction forces were recorded at various displacement values using a surface micro-machined force gauge [26. Three sets of experimental data were taken, each representing an FCBM of identical geometry, but located at alternate locations on the same SUMMiT V die. The force measurements were limited to displacements approaching $F_{\max }$ on the forcedisplacement plot when displacing the FCBM from the first stable equilibrium position to the second, and approaching $F_{\min }$ when displacing the FCBM from the second to the first stable equilibrium position. Because the FCBM transitioned to the alternate stable position after reaching $F_{\max }$ or $F_{\min }$, several reaction forces approaching $F_{\max }$ and $F_{\min }$ were recorded, but few values could be recorded between $F_{\max }$ and $F_{\min }$.

Figure 12 compares the model predictions to the average experimental data [19, 27. A comparison of $F_{\max }$ and $F_{\min }$ results are listed in Table 4 Each of the models matched the region to the left of $F_{\max }$ on the force-displacement plot well. The 
Table 3. Geometry of the SUMMiT V device design.

\begin{tabular}{|c|c|}
\hline Variable Description & Magnitude \\
\hline AL1 & $13.7 \mu \mathrm{m}$ \\
\hline AL2 & $13.7 \mu \mathrm{m}$ \\
\hline RAL & $103.3 \mu \mathrm{m}$ \\
\hline AW & $1.4 \mu \mathrm{m}$ \\
\hline RAW & $6.2 \mu \mathrm{m}$ \\
\hline THETA & $2.44^{\circ}$ \\
\hline PHI & $2.44^{\circ}$ \\
\hline ALPHA & $2.44^{\circ}$ \\
\hline T & $4.75 \mu \mathrm{m}$ \\
\hline EX & $164 \mathrm{GPa}$ \\
\hline PR & 0.23 \\
\hline YDISP & $11 \mu \mathrm{m}$ \\
\hline
\end{tabular}

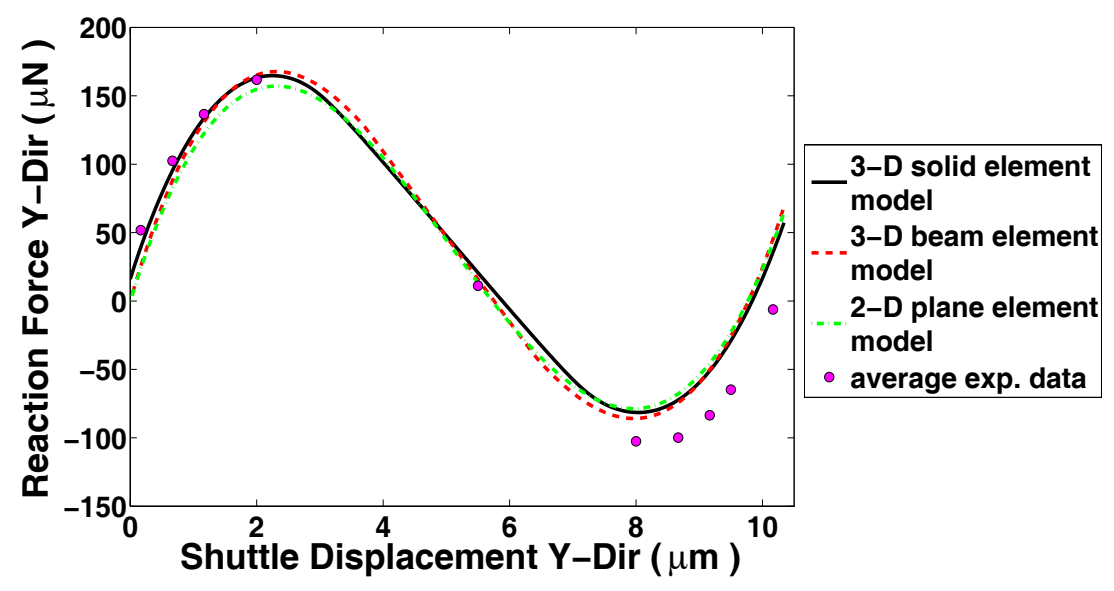

Figure 12. Model comparison to experimental data 19, 27] for the SUMMiT V device.

models underestimated the value of $F_{\min }$ and the region to the right of $F_{\min }$ slightly, but followed the trend well. Overall, each of the models proved to accurately predict the force-displacement relationship of the FCBM.

\section{Predicting Experimental Data for the MUMPs Device}

The validated models were used to reproduce the two drastically different behaviors retrieved from the MUMPs device. Two FCBMs with the same material properties and dimensions were tested at different times after being released. Figure 13 shows that each of the models could accurately produce the first of the two behavior conditions (Device 1). This was expected because the 2-D models were already able to predict these results. However, Figure 14 shows that the 3-D solid element model was able to predict results similar to the Device 2 behavior, while the 2-D plane element model was not. The 3-D beam element model produced results similar to the 3-D solid element 
Table 4. Comparison of $F_{\max }$ and $F_{\min }$ for model validation.

\begin{tabular}{|c|c|c|c|}
\hline Model & $\begin{array}{c}\text { Exp. } \\
F_{\max } / F_{\min } \\
(\mu N)\end{array}$ & $\begin{array}{c}\text { Pred. } \\
F_{\max } / F_{\min } \\
(\mu N)\end{array}$ & $\begin{array}{c}\text { \%Error } \\
F_{\max } / F_{\min }\end{array}$ \\
\hline 2-D PLANE & $163.2 /$ & $154.8 /$ & $-5.4 /$ \\
ELEMENT & -99.07 & -78.67 & 25.9 \\
\hline 3-D BEAM & $163.2 /$ & $165.2 /$ & $1.2 /$ \\
ELEMENT & -99.07 & -85.87 & 15.4 \\
\hline 3-D SOLID & $163.2 /$ & $163.2 /$ & $0.0 /$ \\
ELEMENT & -99.07 & -81.44 & 21.6 \\
\hline
\end{tabular}

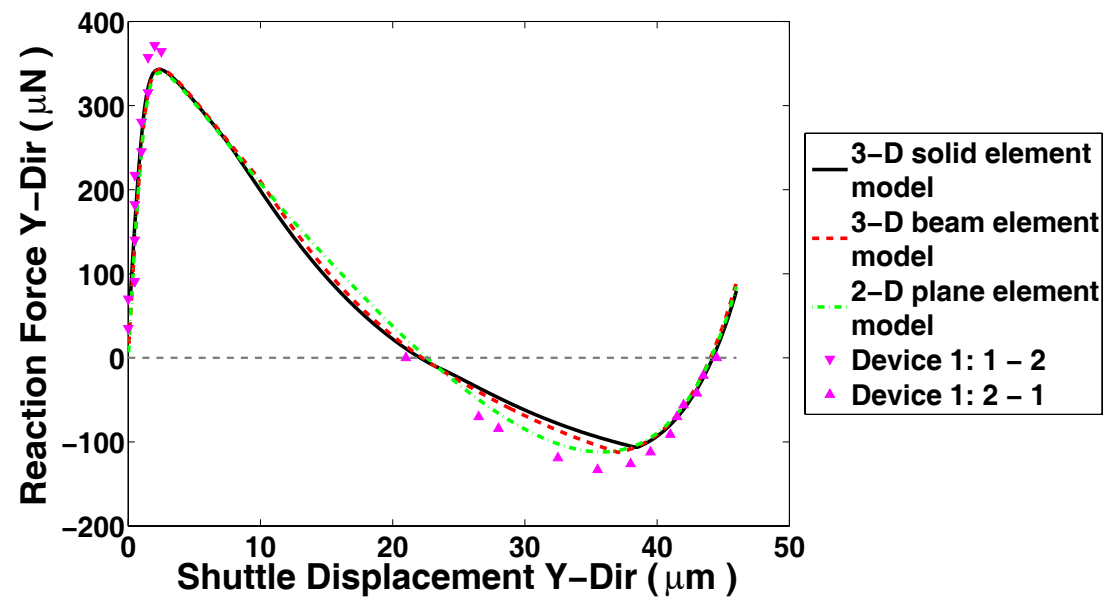

Figure 13. Validation of models using MUMPs Device 1 experimental data.

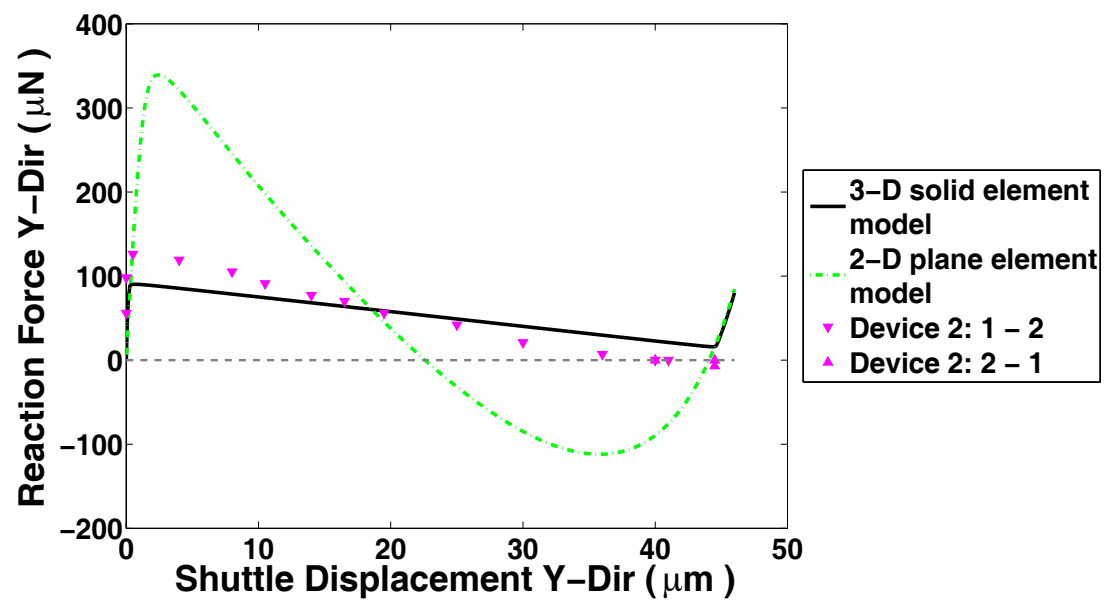

Figure 14. Validation of models using MUMPs Device 2 experimental data.

model. 


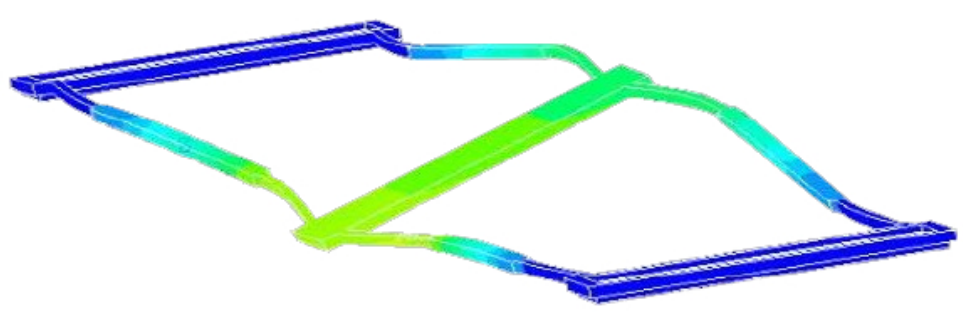

Figure 15. The Behavior $1 \mathrm{FCBM}$ predictions when displaced $23 \mu \mathrm{m}$. The contours represent out-of-plane displacement. The motion is characterized by a larger pitch than Behavior 2, but low roll and yaw.

The ability of the 3-D solid element model to predict Device 2 behavior was a result of analyzing eccentric loading conditions and off-axis forces. Several locations on the FCBM shuttle were chosen for off-axis force application. The results shown in Figure 14 were a result of displacing a node on the back face of the shuttle, and including an off-axis force perpendicular to the top surface of the shuttle, located at the shuttle's center of mass. These findings led to interesting discoveries about the behavior of FCBMs. The results for each device are discussed next.

\section{Discussion of Results}

\subsection{Device 1 - Behavior 1}

Overall, model predictions agreed with the experiment in both trend and magnitude for Device 1. Behavior similar to the force-displacement plot predicted by the 3D models will be referred to as Behavior 1. An FCBM displaying typical Behavior 1 results after being displaced $23 \mu \mathrm{m}$ is shown in Figure 15 . When displaced this distance, Behavior 1 results predicted the largest pitch, and nearly zero roll or yaw. The measured forces are still slightly larger than the predicted forces, and there are several possible causes. First, the material properties of the physical FCBM differs from the FEA model slightly. If the modulus of elasticity in the model were smaller than the actual value, the model predictions would produce smaller reaction forces. Additionally, the geometry of the physical FCBM may slightly differ from the geometry defined in the models. Any one of the dimensions used to fully define the FCBM in the model may be slightly inaccurate due to uncertainties in the manufacturing process [27. Friction between the FCBM shuttle and the substrate could also be a contributing factor.

The inability of the shuttle to roll, pitch, or yaw will affect the experimental force-displacement values. It was hypothesized that the coupler mechanism was slightly constraining the FCBM shuttle from this type of motion, thus increasing $F_{\min }$. To test this hypothesis, the 3 -D solid element model was modified to prescribe the displacement at each node on the front face of the shuttle rather than a single node (thus constraining the roll, pitch, and yaw of the shuttle). This force-displacement relationship, and the experimental data for Behavior 1 is provided in Figure 16. By constraining the shuttle from roll, pitch, or yaw, $F_{m i n}$ increased. Though the coupling mechanism provides some freedom for out-of-plane motion, it does not allow the shuttle to freely roll, pitch, or yaw as it displaces. Therefore, it is plausible that the coupling mechanism is influencing the force-displacement data by smoothing out and increasing 


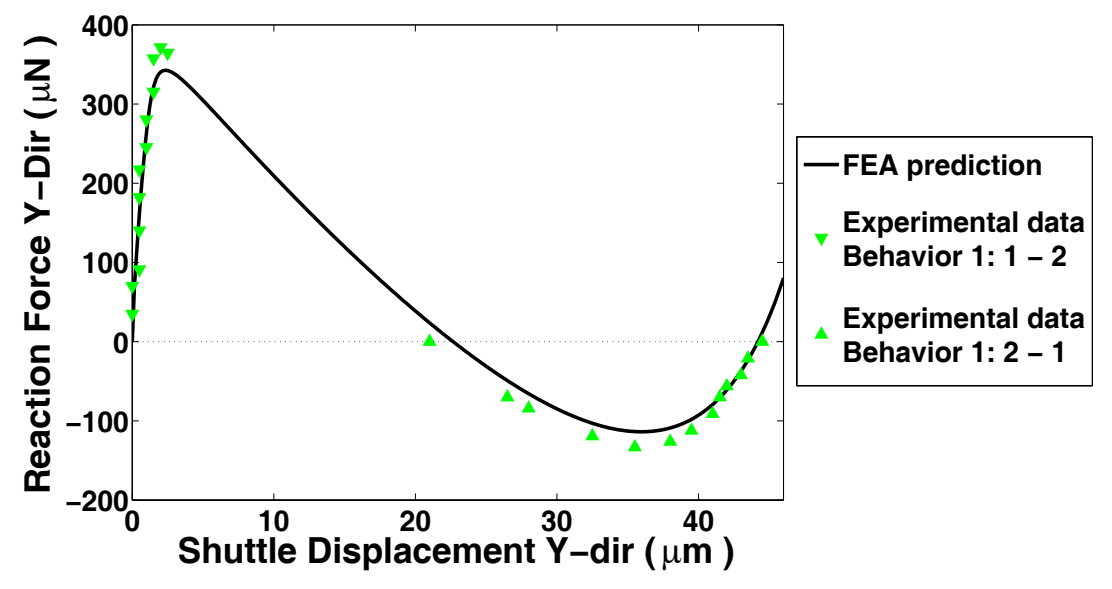

Figure 16. Effect of applying displacement at the face of the shuttle to simulate the coupling mechanism.

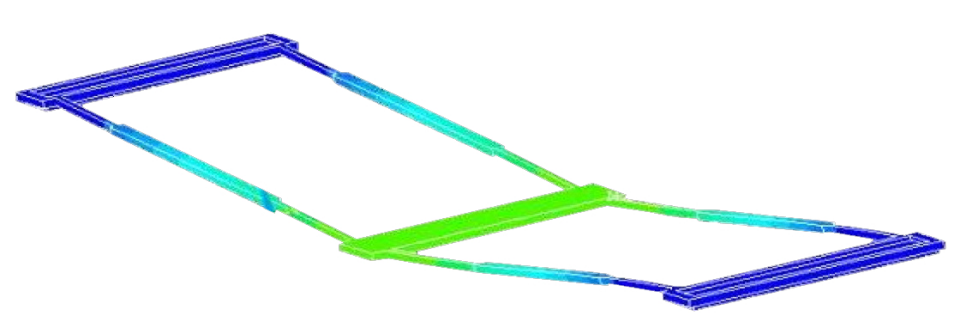

Figure 17. The Behavior $2 \mathrm{FCBM}$ predictions when displaced $23 \mu \mathrm{m}$. The contours represent out-of-plane displacement. The motion is characterized by larger Z-displacements than Behavior 1.

the magnitude of the $F_{\min }$ region.

\subsection{Device 2 - Behavior 2}

The behavior similar to the force-displacement plot associated with Device 2 will be referred to as Behavior 2. The results of the experiment showed a radical unexpected departure from the plot associated with Device 1. This force-displacement plot has much lower peak reaction forces, and is nearly linear between $F_{\max }$ and $F_{\min }$. The results initially appeared to follow the plot produced by Device 1, but strayed from the expected path before reaching the expected $F_{\max }$. Though the reaction forces were greatly reduced, the force-displacement plot still suggested that the mechanism was bistable. An FCBM displaying typical Behavior 2 results after being displaced 23 $\mu \mathrm{m}$ is shown in Figure 17. When displaced this distance, Behavior 2 results predicted nearly zero roll, pitch, or yaw, and larger Z-displacements.

It was hypothesized that the cause of such characteristics is due to the threedimensional effects associated with the FCBMs. Many of the past 2-D models assumed that perfect symmetry was associated with the FCBM geometry and applied force location. However, the FCBM shuttle is free to roll, pitch, and yaw as it is moving to 
its alternate stable equilibrium position. The FCBM's ability to displace out-of-plane can also affect the behavior of the mechanism, including the reaction forces of the force-displacement plot.

The ability of the 3-D solid element model to predict Behavior 2, while the 2-D model was not able to do so suggests that the hypothesis was correct. The 3-D solid element model is capable of incorporating the non-linear three-dimensional behavior of FCBMs in the analysis. To produce Behavior 2 results, off-axis forces were required during analysis. These off-axis forces caused differing roll, pitch, and yaw results. Behavior 2 trends include reduced peak reaction forces, and a nearly linear region in the force-displacement curve. The discrepancy between the experimental data and model prediction are the result of three-dimensional effects. This was further investigated by exploring 3-D models with various loading conditions [21, 20].

\section{Conclusion}

The results in this paper suggest that the difference in Behavior 1 and Behavior 2 is caused by three-dimensional effects that were not accounted for in 2-D models. The 3 -D solid element model was able to predict the Behavior 1 and Behavior 2 results experienced in laboratory testing. Behavior 1 occurred when no off-axis or eccentric loads were applied, or when the magnitude of those forces were lower. Behavior 2 occurred when larger off-axis or eccentric loading conditions occurred. The model can accurately retrieve the reaction forces, displacements of critical nodes, roll, pitch, yaw, and Z-displacement of the shuttle. The efficiencies of the 2-D models are still attractive for cases when they are applicable and when convergence time is critical, such as when many analysis iterations are required in optimizing performance for a particular application. It may be possible to construct the FCBM to isolate it from the three-dimensional effects, such as using stops, large out-of-plane thickness, or using robust design methods [27] to minimize the three-dimensional effects. The possibility of a dramatic difference in behavior for different phenomenon conditions suggest that the effects must be considered and accounted for in FCBM designs.

\section{Acknowledgments}

Funding from Sandia National Laboratories (SNL) via a fellowship through the National Physical Science Consortium (NPSC) is gratefully acknowledged. Support of the National Science Foundation (NSF) through grant number CMI-0428532 is also acknowledged. Thanks also to the Fulton Super Computing center at Brigham Young University for the use of their facilities.

\section{References}

[1] Howell, L., 2001. Compliant Mechanisms. Wiley.

[2] Jensen, B. D., Parkinson, M. B., Kurabayashi, K., Howell, L. L., and Baker, M. S., 2001. "Design Optimization of a Fully-Compliant Bistable Micro-Mechanism." In Proc. ASME IMECE 2001, Vol. 2, pp. 2931 - 2937 New York, NY, United States, Nov 11 - 16, 2001.

[3] Hyman, D., and Mehregany, M., 1999. "Contact Physics of Gold Microcontacts for MEMS Switches." IEEE Transactions on Components and Packaging Technologies, 22(3), pp. 357 -364 .

[4] Qiu, J., Lang, J. H., and Slocum, A. H., 2004. "A Curved-Beam Bistable Mechanism.” Journal of Microelectromechanical Systems, 13(2), pp. 137 - 146. 
[5] Qiu, J., 2003. "An Electrothermally-Actuated Bistable MEMS Relay for Power Applications." PhD thesis, Massachusetts Institute of Technology.

[6] Gomm, T., Howell, L. L., and Selfridge, R. H., 2002. "In-Plane Linear Displacement Bistable Microrelay." Journal of Micromechanics and Microengineering, 12(3), pp. $257-264$.

[7] Luharuka, R., and Hesketh, P. J., 2007. "Design of Fully Compliant, In-Plane Rotary, Bistable Micromechanisms for MEMS Applications." Sensors and Actuators, A: Physical, 134(1), pp. $231-238$.

[8] Sonmez, U., 2007. "Compliant MEMS Crash Sensor Designs: The Preliminary Simulation Results." Intelligent Vehicles Symposium, pp. 303-308.

[9] Hwang, I.-H., Shim, Y.-S., and Lee, J.-H., 2003. "Modeling and Experimental Characterization of the Chevron-Type Bi-Stable Microactuator." Journal of Micromechanics and Microengineering, 13(6), pp. $948-954$.

[10] Tsay, J., Su, L.-Q., and Sung, C.-K., 2005. "Design of a Linear Micro-Feeding System Featuring Bistable Mechanisms." Journal of Micromechanics and Microengineering, 15(1), pp. 63 70.

[11] Wilcox, D. L., and Howell, L. L., 2005. "Fully Compliant Tensural Bistable Micromechanisms (FTBM)." Journal of Microelectromechanical Systems, 14(6), pp. 1223 - 1235.

[12] Anderson, J. K., Howell, L. L., Wittwer, J. W., and McLain, T. W., 2006. "Piezoresistive Sensing of Bistable Micro Mechanism State." Journal of Micromechanics and Microengineering, 16(5), pp. $943-950$

[13] Vangbo, M., 1998. "Analytical Analysis of a Compressed Bistable Buckled Beam." Sensors and Actuators, A: Physical, 69(3), pp. $212-216$.

[14] Vangbo, M., and Bäcklund, Y., 1998. "A Lateral Symmetrically Bistable Buckled Beam." Journal of Micromechanics and Microengineering, 8, pp. $29-32$.

[15] Giannopoulos, G., Monreal, J., and Vantomme, J., 2007. "Snap-Through Buckling Behavior of Piezoelectric Bimorph Beams: I. Analytical and Numerical Modeling." Smart Materials and Structures, 16(4), pp. 1148 - 1157.

[16] Giannopoulos, G., Monreal, J., and Vantomme, J., 2007. "Snap-Through Buckling Behavior of Piezoelectric Bimorph Beams: II. Experimental Verification." Smart Materials and Structures, 16(4), pp. 1158 - 1163.

[17] Anderson, J. K., 2005. "Piezoresistive Sensing of Bistable Micro Mechanism State." Master's thesis, Brigham Young University, Provo.

[18] Koester, D., Cowen, R. M., and Hardy, B., 2002. PolyMUMPs Design Handbook MEMSCAP, Bernin, Revision 8.0.

[19] Wittwer, J., 2005. "Simulation Based Design Under Uncertainty For Compliant Microelectromechanical Systems." PhD thesis, Brigham Young University.

[20] Cherry., B. B., 2008. "Characterizing the Three-Dimensional Behavior of Bistable Micromechanisms." Master's thesis, Brigham Young University.

[21] Cherry, B., and Howell, L., 2008. "Three-Dimensional Behavior of Fully Compliant Bistable Micromechanisms." In Proceedings of the ASME 2008 International Design Engineering Technical Conferences and Computers and Information in Engineering Conference, DETC 2008-49525.

[22] Liu, C., 2006. Foundations of MEMS. Pearson/Prentice Hall.

[23] Johnstone, R., Sameoto, D., and Parameswaran, M., 2006. "Non-Uniform Residual Stresses for Parallel Assembly of Out-of-Plane Surface-Micromachined Structures." Journal of Micromechanics and Microengineering, 16(11), pp. 17-22-.

[24] Kuo, J.-N., Lee, G.-B., and Pan, W.-F., 2004. "Stress-Induced Bending of Micromachined Bilayer Cantilever and its Optical Application." pp. $290-295$.

[25] Mems Device and Reliability Physics Department, Microelectronics Development Laboratory, Sandia National Laboratories, 2005. SUMMiT V - Five Level Surface Micromachining Technology Design Manual., 1st ed. P.O. Box 5800, Albuquerque, NM 87185, 22 September.

[26] Wittwer, J. W., Gomm, T., and Howell, L. L., 2002. "Surface Micromachined Force Gauges: Uncertainty and Reliability." Journal of Micromechanics and Microengineering, 12(1), pp. $13-20$.

[27] Wittwer, J. W., Baker, M. S., and Howell, L. L., 2006. "Robust Design and Model Validation of Nonlinear Compliant Micromechanisms." Journal of Microelectromechanical Systems, 15(1), pp. $33-41$. 